\title{
Morphological and dynamical properties of small-scale chromospheric features deduced from IBIS observations
}

\author{
L. Contarino ${ }^{1}$, F. Zuccarello ${ }^{2}$, P. Romano ${ }^{3}$, D. Spadaro ${ }^{3}$, and I. Ermolli ${ }^{1}$
}

\author{
1 INAF - Osservatorio Astronomico di Roma, via Frascati 33, 00040 Monte Porzio Catone, Italy \\ e-mail: 1cont@oact.inaf.it \\ 2 Dipartimento di Fisica e Astronomia - Sezione Astrofisica, Università di Catania, via S. Sofia 78, 95123 Catania, Italy \\ 3 INAF - Osservatorio Astrofisico di Catania, via S. Sofia 78, 95123 Catania, Italy
}

Received 18 May 2009 / Accepted 21 August 2009

\begin{abstract}
Context. In the past, chromospheric features were mostly studied by analyzing observations performed along the $\mathrm{H}_{\alpha}$ profile, but several aspects related to their formation and dynamics remained uncertain and poorly understood. Recently, new investigations have been carried out using data obtained along the Ca II line at $854.21 \mathrm{~nm}$, providing new inputs for clarification of some of these aspects. Aims. In order to give a further contribution to the comprehension of the physical processes occurring in small-scale chromospheric features, we analyzed high spatial and temporal resolution images, acquired along the Ca II $(\lambda=854.21 \mathrm{~nm})$ line with the Interferometric Bidimensional Spectrometer (IBIS). We studied four chromospheric structures such as mottles and arches belonging to an arch filament system (AFS) inside a bipolar region, observed on October 3, 2006.

Methods. We evaluated the plasma velocity along the line of sight (LOS) using two methods: the Doppler shift of the centroid of the line profile and the cloud model. Also, we deduced the mean temperature, the Doppler width, the optical thickness and the source function in the structures to which we could apply the cloud model.

Results. The pattern of the LOS velocity in the four mottles showed different behaviors. A mottle, initially, showed positive and negative velocities in eastern and western endpoints, respectively, then the plasma motion seems to reverse over a period of about $4 \mathrm{mn}$. In another mottle a motion characterized by alternate upward and downward plasma flow along the main axis was recorded. Irregular upward and downward motions along the other two mottles confirm previous results. The LOS velocities measured in the AFS, observed during the decay phase of the region, are of the same order of magnitude as those measured in short-lived active regions during their emergence phase.

Conclusions. The observations carried out in the Ca II line allowed us to obtain information on small-scale magnetic features, like mottles and AFS, observed in the chromosphere. These results, on one hand, confirm previous results obtained using data acquired in the $\mathrm{H}_{\alpha}$ line, and on the other hand, provide new clues to the dynamic similarities between mottles and dynamic fibrils. Moreover, this study allowed us to single out the presence of an AFS during a phase characterized by decreasing magnetic flux and the approach of the opposite polarities.
\end{abstract}

Key words. Sun: chromosphere - Sun: activity

\section{Introduction}

The solar chromosphere is highly inhomogeneous. Its main small scale features, observed in the $\mathrm{H}_{\alpha}$ lines, are jetlike structures named spicules, dynamics fibrils (DFs) and mottles.

Spicules are structures observed at the solar limb with heights of 5000-10000 km and lifetimes of 5-15 mn. They are usually associated with regions of enhanced magnetic flux (Beckers 1972).

DFs are dark structures that are found in active regions. These fibrils are relatively thin $(\sim 100-700 \mathrm{~km})$ and short $(\sim 1250 \mathrm{~km})$ and have lifetimes of 3 to $6 \mathrm{mn}$. The temporal evolution of DFs is dominated by a clear upward and downward motion along the main axis (De Pontieu et al. 2007a). Recent studies have shown that DFs are driven by and can channel photospheric oscillations into the chromosphere and the corona (Hansteen et al. 2006; de Wijn \& De Pontieu 2006; De Pontieu et al. 2007a).

Mottles are elongated dark structures that outline the network boundaries in the quiet Sun. They have widths and heights in the range 500-2000 km and 5000-10000 km, respectively, and have lifetimes of 5-10 $\mathrm{mn}$. Mottle configuration is governed by the local magnetic field and they are usually organized in small groups, called chains, or larger groups, called rosettes. In the former configuration, the mottles point in the same direction, while in the latter a circular structure is observed, with a bright center surrounded by several mottles aligned radially outwards. Strong magnetic flux $\left(\sim 10^{20} \mathrm{Mx}\right)$ was detected in the bright center of rosettes (Dara-Papamargaritis \& Koutchmy 1983).

Several authors evaluated plasma vertical velocities in mottles (Suematsu et al. 1995; Tsiropoula et al. 1994; Tziotziou et al. 2003; Rouppe van der Voort et al. 2007) and found that their footpoints show downflows, while their tops have alternating upflows and downflows. De Pontieu et al. (2007b) found also that mottles show transverse motion in the direction perpendicular to their axis, with an average velocity of $\sim 13 \mathrm{~km} \mathrm{~s}^{-1}$.

Mottles are considered as the principal channel through which mass and energy is supplied from lower layers of the solar atmosphere to the corona and solar wind (Tziotziou et al. 2003; De Pontieu et al. 2007a). Hence, they can make a significant contribution to coronal heating processes.

An arch filament system (AFS) is a bundle of dark arches, crossing the polarity inversion line, that usually appears during 
the emergence of an active region (see, e.g., Spadaro et al. 2004; Zuccarello et al. 2005). The AFSs show a rising velocity at their tops of 10-15 $\mathrm{km} \mathrm{s}^{-1}$ and downflows at their endpoints of 10$50 \mathrm{~km} \mathrm{~s}^{-1}$, have lengths of $1-3 \times 10^{4} \mathrm{~km}$ and a lifetime of the order of $20 \mathrm{mn}$ (Shibata et al. 1989; Yoshimura \& Kurokawa 1999).

Despite the different nomenclature, there are strong indications that these fibrilar fine structures are physically closely related each other and that the key element of all these phenomena is the magnetic field, distributed in different scales over the solar disk (Tsiropoula et al. 1994; Christopoulou et al. 2001; Tziotziou et al. 2004; Rouppe van der Voort et al. 2007).

Moreover, recent studies showed that dynamic fibrils form by chromospheric shock waves occurring when convective flows and global oscillations leak into the chromosphere along the magnetic field lines. The mechanism forming the mottles in the quiet Sun is most likely the same that produces fibrils in active regions, even if it might be modified by the weaker magnetic fields (De Pontieu et al. 2007a; Rouppe van der Voort et al. 2007).

In spite of the advances made in recent years both in observations and in simulations, the diagnostics and dynamics of small-scale chromospheric structures, the determination of several thermodynamic parameters and the mechanisms responsible for their formation are still poorly understood. This is also due to the limited number of high resolution observations: the spatial scale of these features, of a few hundred km, and their lifetime, of a few minutes, are very close to current observational limits. The advanced image processing techniques adopted in the analysis of observations collected by the new generation of ground-based telescopes and space missions, together with new 3-D simulations (Leenaarts et al. 2009), are expected to provide us the possibility to fill the current gaps.

In this context, we analyzed a high spatial and temporal resolution series of images acquired along the Ca II $(\lambda=854.21 \mathrm{~nm})$ line with the Interferometric Bidimensional Spectrometer (IBIS, Cavallini 2006), installed at the Dunn Solar Telescope of the US National Solar Observatory. We investigated the morphological and dynamical properties of dark chromospheric structures (mottles and AFS) inside a bipolar region in its decay phase.

\section{Observations and data reduction}

\subsection{Data set}

We analyzed data acquired during an observational campaign performed on October 2-8, 2006 at the Dunn Solar Telescope (DST) of the US National Solar Observatory at Sac Peak, using the Interferometric Bidimensional Spectrometer (IBIS, Cavallini 2006). Data were acquired with a high order adaptive optics system (Rimmele 2004).

In this study, we analyze a small magnetic region observed at the center of the solar disk $(7.2 \mathrm{~N}, 3.1 \mathrm{~W})$ on October, 3 .

The full dataset consists of 120 sequences along the profiles of the Fe I line ( $\lambda=709.0 \mathrm{~nm}, 29$ spectral points $)$ and the Ca II line $(\lambda=854.21 \mathrm{~nm}, 39$ spectral points), acquired in the time interval 15:52-16:43 UT. At the same time, broad band images centered at $\sim 700.0 \mathrm{~nm}$ and G-band images were obtained. The images were acquired in binned mode with $512 \times 512$ pixels, with a pixel scale of 0.17 arcsec (corresponding to $\sim 120 \mathrm{~km}$ at the solar surface), and an exposure time of $20 \mathrm{~ms}$. The images have a circular field of view of 80 arcsec in diameter. For our analysis, we selected only eight spectral scans from the entire observational sequence, characterized by the best quality. Then, we selected two square fields of view of $56 \times 56$ arcsec on each image of this dataset. The time interval between two successive full spectral scans is $\sim 20 \mathrm{~s}$ ( $\sim 3$ spectral points per second).

Data were reduced using the standard IBIS pipeline (Janssen \& Cauzzi 2006), which works by steps and corrects the images for CCD non linearity effects, dark current, gain and flat-field. We also corrected for the systematic blueshift of the instrumental profile when moving from the optical axis toward the edges of the field of view.

To study the magnetic evolution of the target, we analyzed a sequence of full-disk line of sight magnetograms acquired by MDI/SOHO every $96 \mathrm{mn}$ from 1 October at 09:35 UT to 4 October at 17:36 UT. For each magnetogram, we considered a subfield of $128 \times 128$ pixels $\left(\sim 170 \times 170 \mathrm{Mm}^{2}\right)$ containing the target. One MDI/SOHO high resolution magnetogram, acquired at 13:13 UT, was also used in our study. The data analyzed were compensated for MDI linear and non-linear response (Berger \& Lites 2002) and for projection effects (Freeland \& Handy 1998).

\subsection{Global morphology}

The target is a magnetic bipolar region with a lifetime of 4 days, without a NOAA identification number. It can be considered an intermediate structure between an ephemeral region and a shortlived active region.

Figure 1 shows the data analyzed in this study. In particular, Fig. 1a shows a G-band image (obtained using the multi-object multi-frame blind deconvolution image restoration technique (MOMFBD, van Noort et al. 2005)) and Fig.1b gives an image from the data taken in the blue wing of Ca II $(\Delta \lambda=-0.02 \mathrm{~nm})$. Figure 1c shows an example of images acquired in the center of the $\mathrm{Ca}$ II line. In Fig.1d a high resolution magnetogram acquired by $\mathrm{SOHO} / \mathrm{MDI}$ is reported.

The target appears as a bright region in corona (from inspection of full disk SOHO/EIT images). In the Ca II data the region consists of several dark mottles, surrounding a central part with two bright knots, each having a diameter of about 10 arcsec, connected by several arch filaments (AFs). The target seems therefore to have a rosette configuration (Fig. 1c). Moreover, the AFS is visible in the decay phase of the region.

Analysis of full-disk magnetograms shows that (Fig. 2a-d) the region is characterized by an increasing separation of the opposite polarities (their distance increases from 22 to 25 arcsec, Fig. 2a,b), during the first two days of its evolution, while these polarities approach each other (their distance becomes 13 and then 9 arcsec, respectively, Fig. 2c-d) during the following two days. As shown in the following, the approaching phase corresponds to the decay phase.

We evaluated the positive and negative magnetic flux, considering a threshold of 80 Gauss to exclude background effects. Figure 2e shows the evolution of negative (diamonds) and positive (asterisks) magnetic flux. Vertical black lines mark the end of each day and the red line indicates the time of IBIS observations. The first evidence of the magnetic flux emergence is recorded on the 1st of October, at 09:35 UT; the maximum flux is recorded on the second day, and then it decreases until vanishing on October, 4.

\subsection{Data analysis}

For each sequence of data taken around the Ca II line, we reconstructed the line profile in each image pixel by fitting the 


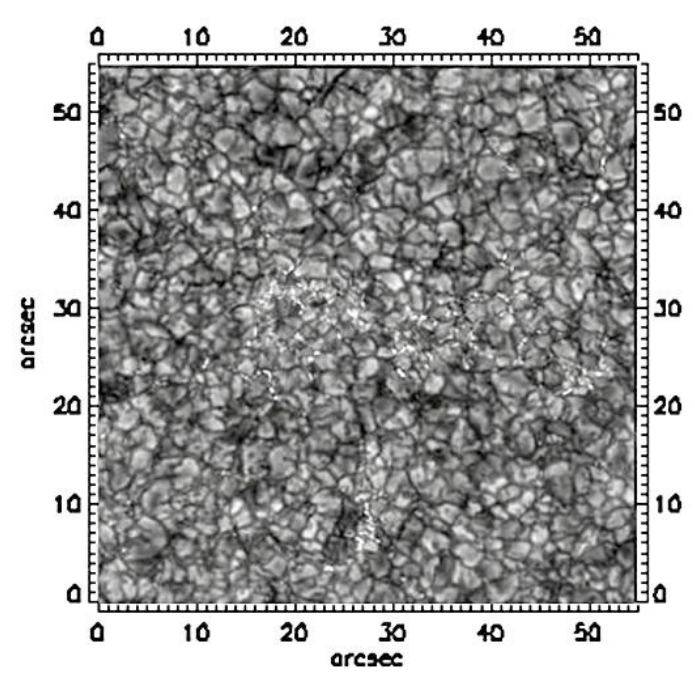

(a)

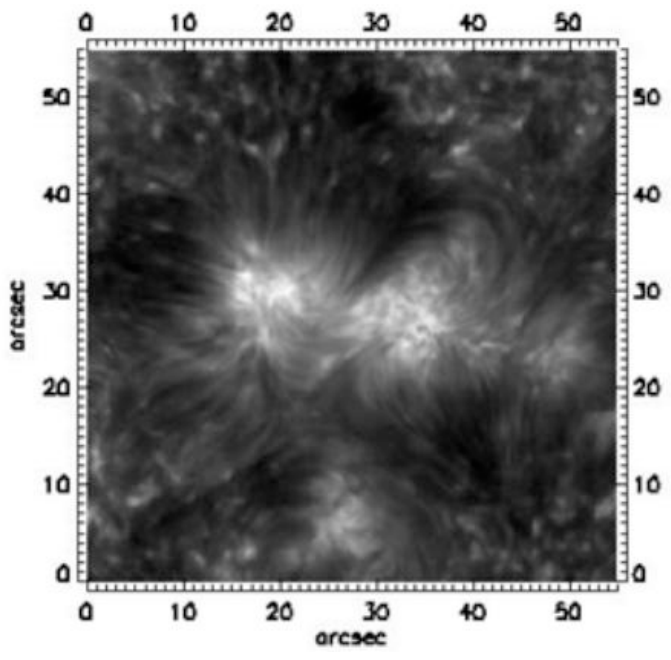

(c)

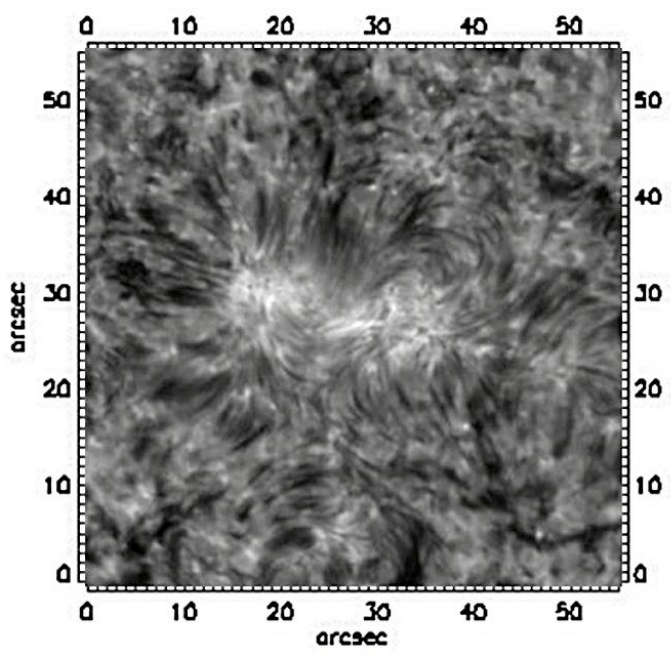

(b)

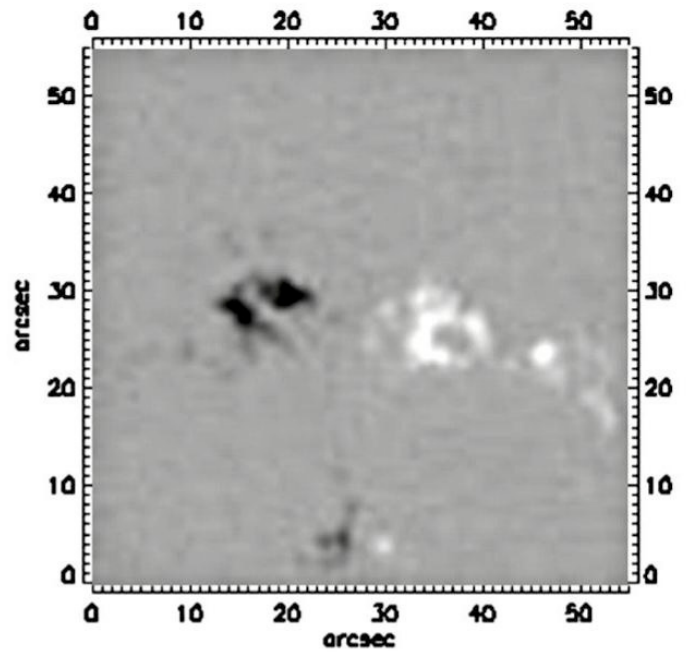

(d)

Fig. 1. a)-c) Sequence of images of the target from the data acquired at 15:52:51 UT in: a) G-band channel; b) blue wing of the Ca II ( $\Delta \lambda=$ $-0.02 \mathrm{~nm})$; c) Ca II line center (854.21 nm); d) high resolution magnetogram acquired by SOHO/MDI at 13:13 UT. In all images the field of view is $56 \times 56 \operatorname{arcsec}\left(\sim 39200 \times 39200 \mathrm{~km}^{2}\right)$. North is on the top, West on the right.

corresponding signals obtained in the monochromatic images with a linear background and a Gaussian shaped line:

$I_{j k}=A_{0} \exp \left(-\left(\frac{\lambda_{k}-A_{1}}{A_{2}}\right)^{2}\right)+A_{3}+A_{4} \lambda_{k}$

where $I_{j k}$ is the observed count rate in pixel $j$ at wavelength $\lambda_{k}$, $A_{0}$ the height of the Gaussian, $A_{1}$ the centroid wavelength of the Gaussian, $A_{2}$ the $1 / \mathrm{e}$ half width of the Gaussian, $A_{3}$ the constant term and $A_{4}$ the linear term. Values of the parameters $A_{0}, A_{1}, A_{2}$, $A_{3}$ and $A_{4}$ were obtained by applying the maximum likelihood method. Figure 3 shows an example of the line fit. The Gaussian shaped line satisfactorily reproduces the core of the observed line profile (triangles).

Then, we measured the values of velocity along the line of sight (LOS), by using two methods: 1) the classical method based on Doppler shift of the centroid of the line profiles in each spatial point with respect to the median of the centroid in a 'quiet' sub-field and 2) the cloud model method (Beckers 1964). This method considers the structures as a cloud lying between the observer and an uniform atmosphere described by a reference background (find description of the method and details of our application in Appendix A). The model works quite well for optically thin structures and is useful to study the properties and dynamics of various chromospheric cloud-like structures.

For the Doppler shift measurements, we used as wavelength reference the median of the centroid computed over a sub-field $(200 \times 80$ pixels) in the quiet Sun (see the red box in Fig. $4 a)$.

To estimate the uncertainty in the velocity measurements, we considered the standard deviation of the centroid of the line profile in all points of the field of view (Spadaro et al. 2004) for the Doppler shift method, getting an error of $1.2 \mathrm{~km} \mathrm{~s}^{-1}$. As for the cloud model, assuming an uncertainty of the contrast at the center line of 0.05 , we estimated an error of $0.9 \mathrm{~km} \mathrm{~s}^{-1}$, according to the error propagation method.

\section{Results}

Over the whole field of view we selected two sub-fields where mottles and AFS structures are clearly visible. In particular, subfield $1(\sim 25 \times 25 \operatorname{arcsec})$ shows a well ordered pattern of fine elongated dark mottles and sub-field $2(\sim 30 \times 24$ arcsec $)$ shows an AFS (see Fig. 4b,c). The mottles and the AFS are identified by visual inspection of the intensity time series and their axes 


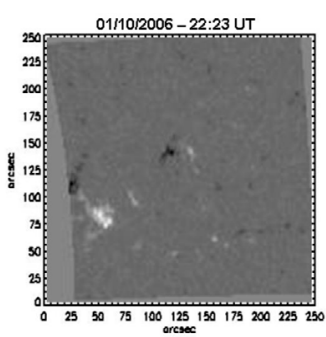

(a)

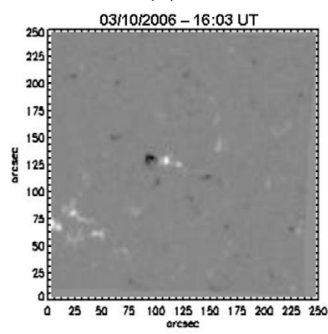

(c)

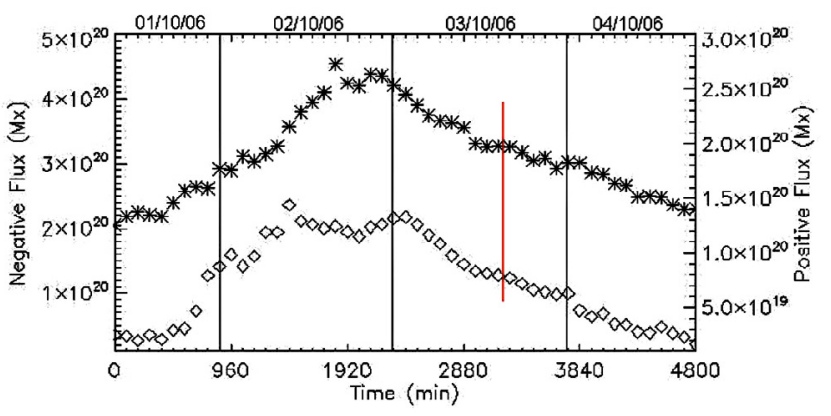

(e)

Fig. 2. a)-d) Sequence of magnetograms acquired by MDI/SOHO. North is on the top, West on the right; e) negative (diamonds) and positive (asterisks) magnetic flux from 09:35 UT, on October 1st to 17:36 UT, on October 4th, 2006, as deduced from MDI full-disk magnetograms. Vertical black lines mark the end of each day and the red line indicates the time of IBIS observations.

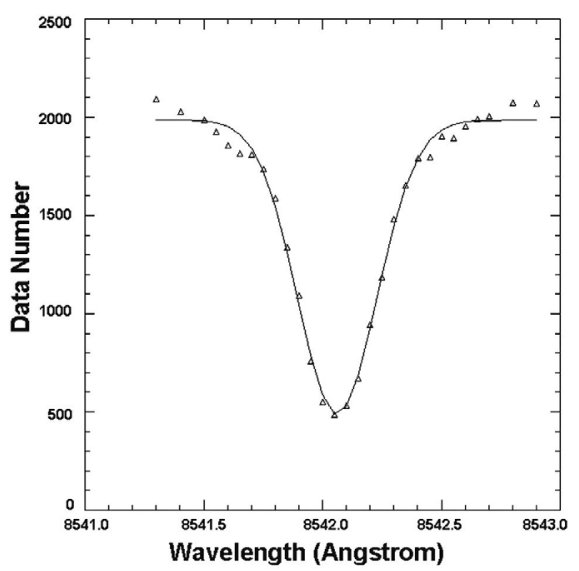

Fig. 3. An example of observed line profile (triangles) with a Gaussian fit (solid line).

and paths, respectively, were traced accordingly, following the behaviour of the intensity signal (Fig. 4d,e).

Figure 5 shows the negative (blue) and positive (red) magnetic concentrations, deduced from the high resolution magnetogram acquired by MDI at 13:13 UT, overplotted on the image extracted from the data taken at the center of the Ca II line, acquired with IBIS at 15:52:51 UT. We see that the regions where strong magnetic fields (a few hundred Gauss) are measured coincide with the two chromospheric bright regions and that the AFS has both footpoints anchored inside them; conversely, the mottles chosen for our analysis have only the western endpoints inside the negative magnetic concentration.

We applied the classical Doppler shift method to both the sub-fields, while the iterative procedure of the cloud method was applied only to sub-field 1 and to the top of the AFS in subfield 2. In fact, the assumptions of cloud-model can not be applied to the whole sub-field 2 because the ends of the AFS are too close to the two bright knots, so that their emission makes the absorption not sufficient to obtain the necessary contrast with the background. We obtained a 2D map for each parameter of the cloud-model, which shows the behavior of the parameter in the analyzed sub-field.

Moreover, in order to evaluate the thermal state of the analyzed structures, we calculated the temperature along the structures using Eq. (2), which relates the spectral line Doppler width to the plasma temperature:

$\Delta \lambda_{\mathrm{D}}=\lambda_{0} / c \sqrt{\xi_{\mathrm{t}}^{2}+2 K T / m}$

where $m$ is the Ca atom rest mass, $K$ the Boltzmann constant, $c$ the velocity of light, $\xi_{\mathrm{t}}$ the turbulent velocity and $\lambda_{0}$ the central wavelength of the line. To obtain significant (non-negative) values of temperature, we assumed a turbulent velocity $\left(\xi_{\mathrm{t}}\right)$ of 3,4 and $6 \mathrm{~km} \mathrm{~s}^{-1}$, for the eastern top end, the central part and the western footpoint, respectively, of each mottle.

\subsection{Mottles}

We selected four different mottles inside the sub-field 1 , named $a, b, c$ and $d$, that are well separated from each other and have a linear shape (Fig. 4b).

In Fig. 6 we report the histograms of the source function, Doppler width, optical depth and LOS velocity obtained inside the mottles, considering all the values of the parameters derived from the cloud model, for all the selected mottles and times considered in this study. The values for each mottle are extracted over a strip of about 0.68 arcsec centered along the main axis of the structure.

The source function distribution peaks near 0.3, the Doppler width close to $0.015 \mathrm{~nm}$, while the optical depth is around 0.3 0.4 and then rapidly decreases. These values are slightly lower (by a factor of 2-3) than those reported in the literature, as deduced from $\mathrm{H}_{\alpha}$ observations of mottle structures (Tziotziou et al. 2003; Lee et al. 2000), except for the source function, which is a factor of 3 greater.

The velocity distribution is almost symmetric and ranges between -10 and $+10 \mathrm{~km} \mathrm{~s}^{-1}$, with a peak near $+0.3 \mathrm{~km} \mathrm{~s}^{-1}$ (upflow motion). This distribution is an indication of the presence inside mottles of both downward and upward velocities.

Figure 7 shows the LOS velocity maps for the sub-field 1 deduced using the cloud model (a) and the Doppler shift (b); black lines indicate the position of the analyzed structures. Red (blue) areas indicate downflow (upflow) plasma velocity. We found that application of the Doppler shift method to this field of view, yields to the same behavior of the velocity field, but with lower values (by a factor of 2) than those calculated using the cloud model.

We can see that the selected mottles do not show a regular behavior of the LOS velocity, along their length. 


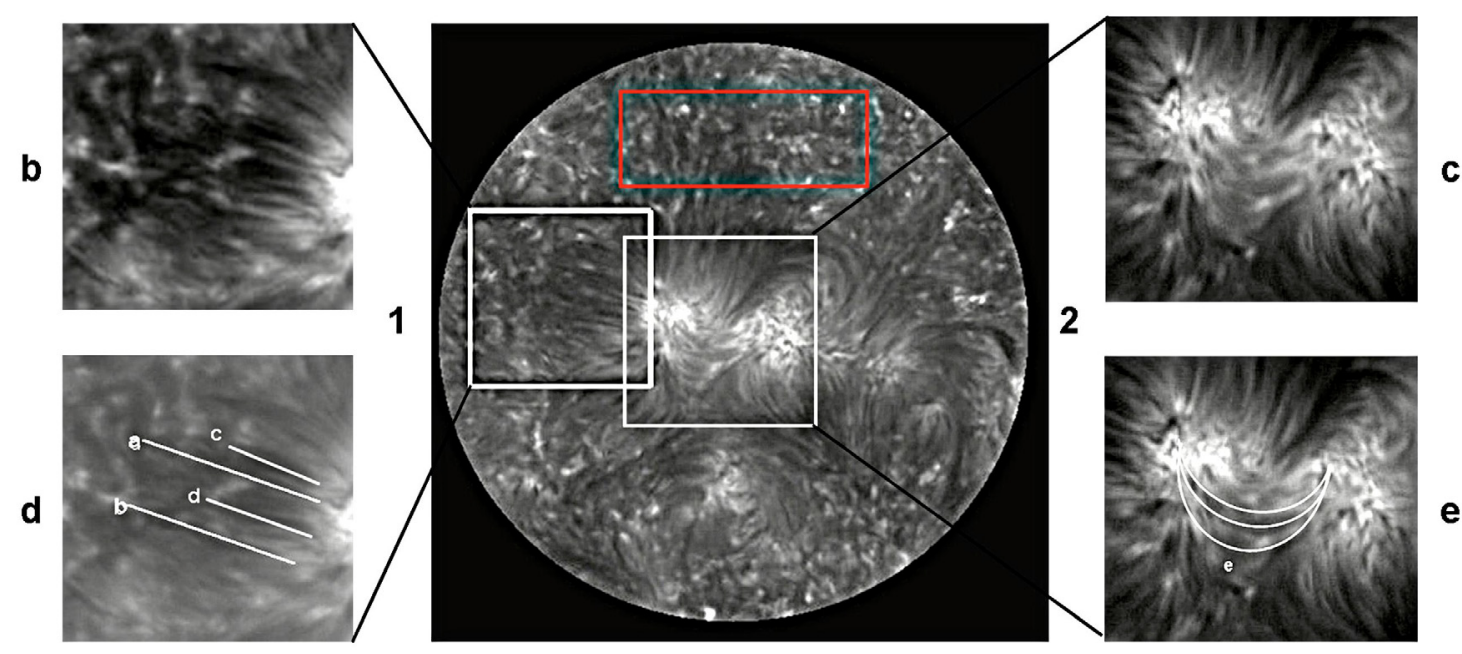

a

Fig. 4. a) Whole field of view of the region observed, acquired by IBIS in the Ca II line $(854.21 \mathrm{~nm})$ at 15:52:51 UT. The red box shows the sub-field where the wavelength reference was computed in order to estimate the Doppler shift. The boxes b)-d)) show the sub-fields analyzed in this study with the corresponding structures labeled (boxes d)-e)) as described in Sects. 3.1 and 3.2.

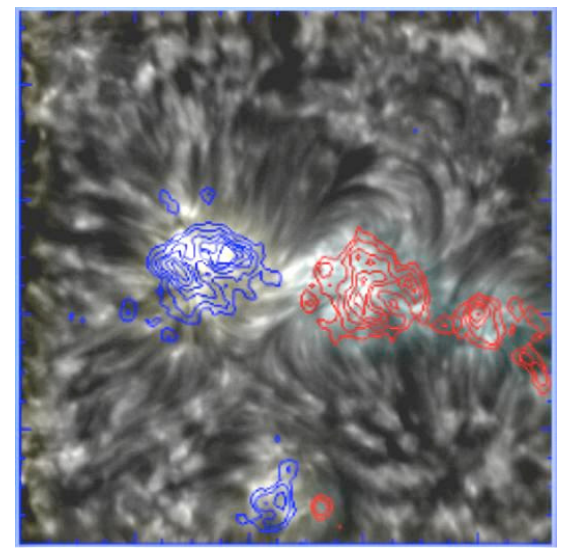

Fig. 5. Positive (red) and negative (blue) magnetic flux contours inferred from MDI high resolution magnetogram, acquired at 13:13 UT, overplotted on the chromospheric image from the data acquired in the center of the Ca II line at 15:52:51 UT.

Therefore, to evaluate the LOS velocities and their evolution along the structures, we considered for each mottle a box of $6 \times$ 3 pixels (corresponding to $1 \times 0.5$ arcsec) near both the western footpoint (WF) and the eastern top end (ET), and of $10 \times 3$ pixels (corresponding to $1.7 \times 0.5 \operatorname{arcsec}$ ) in the central part $(\mathrm{CP})$.

The plots in Fig. 8 summarize the median values of LOS velocity at the ends and center of the mottles $a, b, c$ and $d$. We found that mottle $a$ (with a length of about $11100 \mathrm{~km}$, Fig. 8a) shows nearly oscillating plasma velocities (alternatively upward and downward motions), with a similar behaviour in all the examined segments of the mottle. The only exception occurs near the end of the time sequence, when ET exhibits downward motions, conversely to the upward motions predominantly noticed in the other two segments. Mottle $b$, that has a length of about $10500 \mathrm{~km}$, initially shows positive (upward plasma motion) and negative (downward plasma motion) velocity in the eastern end (blue line in Fig. 8b) and western footpoint (red line in Fig. 8b), respectively. Then, the plasma motion seems to reverse. Its central part has both downflows and upflows (black line in Fig. 8b). The pattern of velocity near the endpoints seems to reflect the oscillatory behavior of $4 \mathrm{mn}$ reported already by other authors (see discussion in Sect. 4). Mottles $c$ and $d$ have a length of about $6000 \mathrm{~km}$, and show both negative and positive velocity at their endpoints and in the central part, without an apparent regularity (see Figs. 8c,d).

Suematsu et al. (2008) analyzed several time series of spicules at the limb and found that they show alternatively upward and downward motions along a rigid path line. The period of oscillations ranges from 1 to $4 \mathrm{mn}$. If mottles and spicules are magnetic features closely related each other, the LOS velocity trends found here could be of the same nature.

The values of the temperature along the mottles, deduced from Eq. (2), are reported in Table 1. They are evaluated considering the mean of the median Doppler widths obtained for each time inside the three boxes defined in the structures (see above). We found that nearly all the mottles exhibit a decrease in temperature from the western footpoint to the eastern top end. The mottles $a$ and $c$ have high values of temperature in the western footpoint (marked with asterisks in Table 1), more similar to the characteristic values of the transition region. These values could be overestimated, due to the choice of a small turbulent velocity and/or to the effect of seeing. It is worth noting that Tziotziou et al. (2007) pointed out that the atmospheric seeing significantly influences the determination of the Doppler width in the cloud model inversion, because it broads the line profile and leads to a higher (not real) value of Doppler width, hence affecting the determination of the temperature.

\section{2. $A F S$}

Figure 9 shows the LOS velocity maps of the sub-field 2, deduced using the Doppler shift of the centroid (b) and the cloud model method (a). Black curves indicate the position of the analyzed AFS (named $e$ ). Red (blue) areas indicate downflow (upflow) plasma velocity.

The AFS, which has an average length of about $15300 \mathrm{~km}$, shows positive velocities in its central part and negative velocities in the footpoints (see Table 2). This indicates upward motion of the body of the structure rising into the chromosphere, and downward plasma motions along the magnetic lines at the footpoints of the AFS. This scenario is in agreement with the 


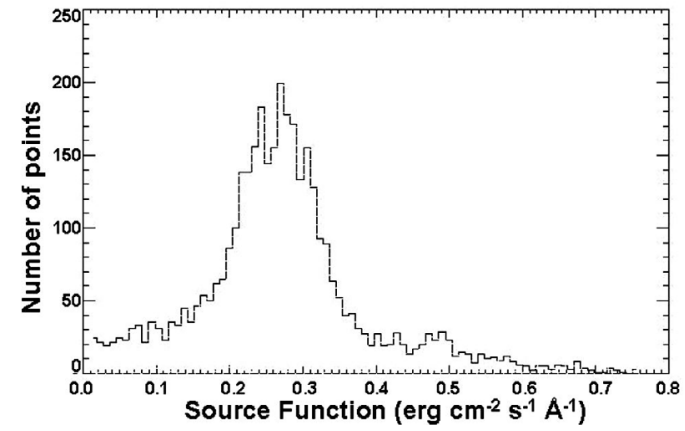

(a)

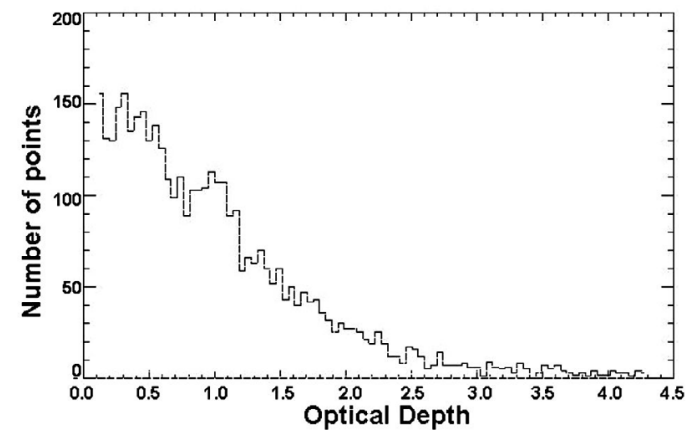

(c)

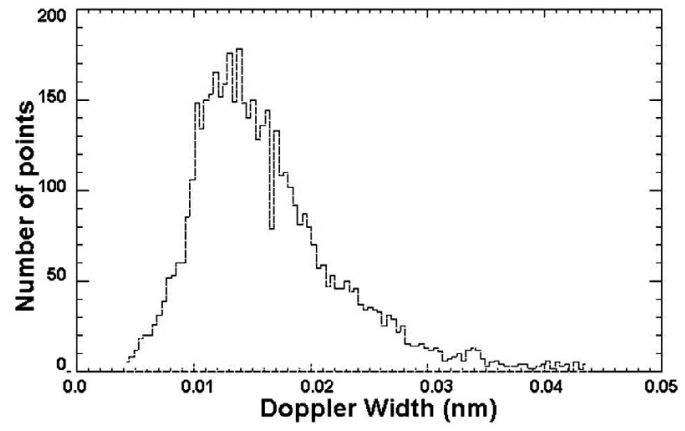

(b)

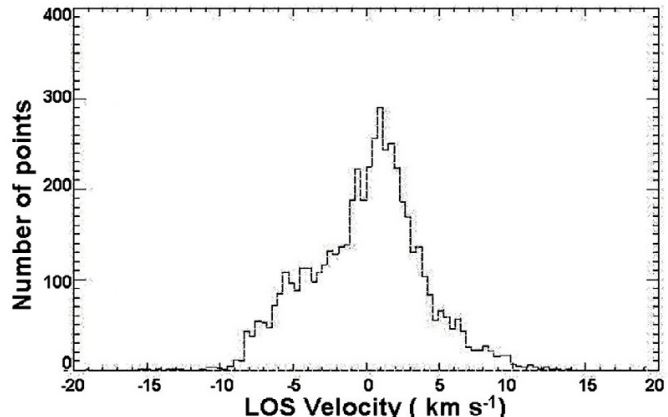

(d)

Fig. 6. Histograms of a) source function, b) Doppler width, c) optical depth and d) LOS velocities deduced with the cloud model for all the mottles and all the times considered in this study.

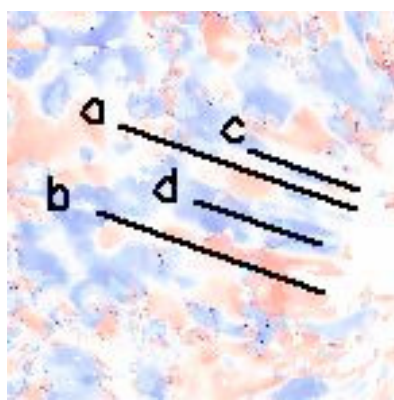

(a)

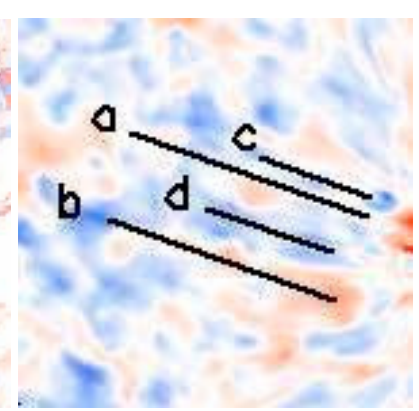

(b)

Fig. 7. LOS velocity maps of the sub-field 1 obtained with cloud model methods a) and Doppler shift b). Blue (red) areas indicate upward (downward) plasma motions. Black lines indicate the analyzed structures.

behavior of the AFS observed in the first evolutionary phase of recurrent and short-lived active regions (Spadaro et al. 2004; Zuccarello et al. 2005).

To better evaluate the LOS velocity and their evolution along the structures, we considered a box of $25 \times 25$ pixels (corresponding to $4.2 \times 4.2$ arcsec) near the footpoints (eastern footpoint (EF) and western footpoint (WF)) and of $35 \times 35$ pixels (corresponding to $5.9 \times 5.9$ arcsec) in the central part $(\mathrm{CP})$. For the AFS the boxes are greater than for mottles because we considered the entire AFS system, and not the single AF. Using only velocity values greater than the error, we determined the median in each part.

We found that the LOS velocity is almost constant in time all over the AFS. In the footpoints it ranges between $-3.0 \mathrm{~km} \mathrm{~s}^{-1}$ and $-1.7 \mathrm{~km} \mathrm{~s}^{-1}$ and in the central part it has a mean value of $+1.5 \mathrm{~km} \mathrm{~s}^{-1}$. These values are similar to those recorded for a short-lived active region in its emergence phase (Zuccarello et al. 2005).

Table 1. Mean of temperature values along the mottles.

\begin{tabular}{lccc}
\hline \hline & \multicolumn{3}{c}{$\begin{array}{c}\text { Temperature } \\
\left(10^{4} \mathrm{~K}\right) \\
( \pm 2)\end{array}$} \\
\hline Structure & ET & CP & WF \\
\hline Mottle $a$ & 2.1 & 2.8 & $11.3^{*}$ \\
Mottle $b$ & 2.1 & 1.9 & 3.1 \\
Mottle $c$ & 3.6 & 2.8 & $16.2^{*}$ \\
Mottle $d$ & 2.8 & 6.8 & 2.0 \\
\hline
\end{tabular}

The results obtained with the cloud method applied to the central part of the AFS are in agreement with those found with the Doppler Shift (Fig. 9a). The behavior of the LOS velocity is the same, but the values are greater by a factor 3-4.

Using the Eq. (2) and assuming a turbulent velocity $\left(\xi_{\mathrm{t}}\right)$ of $7 \mathrm{~km} \mathrm{~s}^{-1}$, we have evaluated a temperature for the AFS of about $8.2 \times 10^{4} \mathrm{~K}$, for the eastern footpoint, and of about $2.5 \times 10^{4} \mathrm{~K}$ for the central part and the western footpoint. The same considerations about the effects of seeing on the determination of spectral line width in mottle structures hold in this case also.

\section{Discussion and conclusions}

In recent years detailed information on solar chromospheric features was obtained using mainly high resolution observations performed along the $\mathrm{H}_{\alpha}$ line profile. In fact, structures like fibrils and mottles rarely have been observed in the $\mathrm{Ca}$ II $\mathrm{H}$ and $\mathrm{K}$ images. Recent work (Reardon et al. 2009) pointed out that the dissimilar chromospheric appearance, at different atmospheric layers, is only due to observational limits and to some peculiarities of the line formation, such as the photoionization-recombination mechanisms (Cauzzi et al. 2008). 


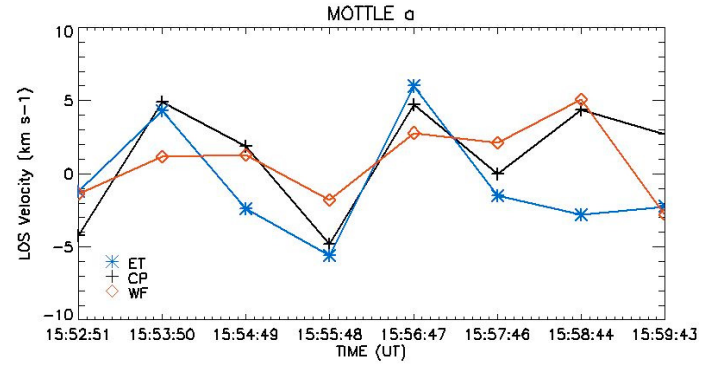

(a)

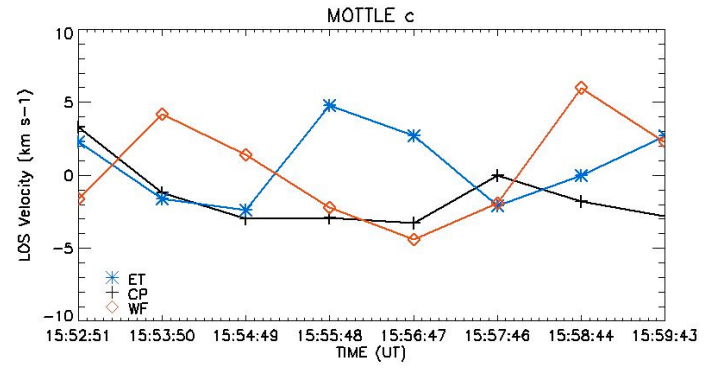

(c)

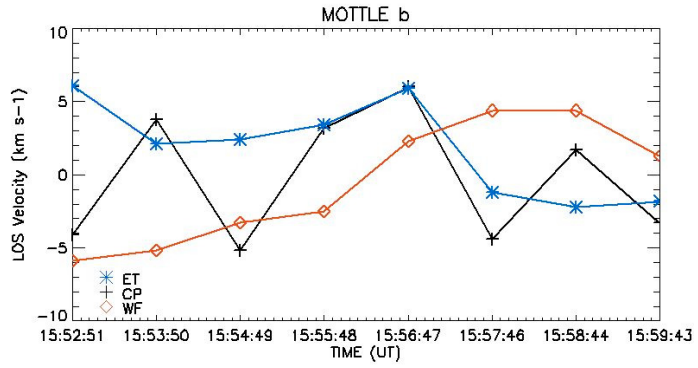

(b)

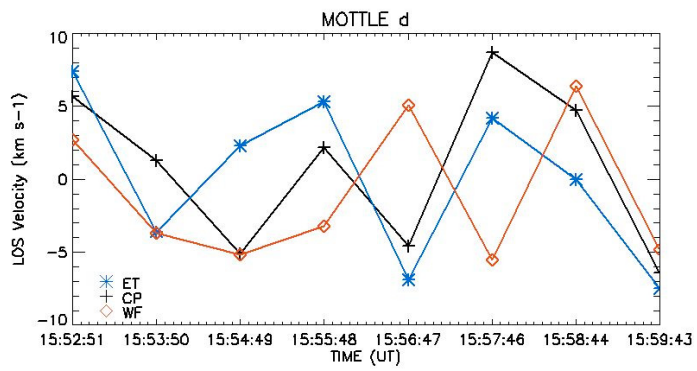

(d)

Fig. 8. a)-d) Plots of the median plasma velocity values along mottles $a, b, c$ and $d$, respectively. In each graph the velocities of the end top $E T$ (asterisk and blue line), the central part $C P$ (plus and black line) and the western footpoint $W F$ (diamond and red line) are plotted.

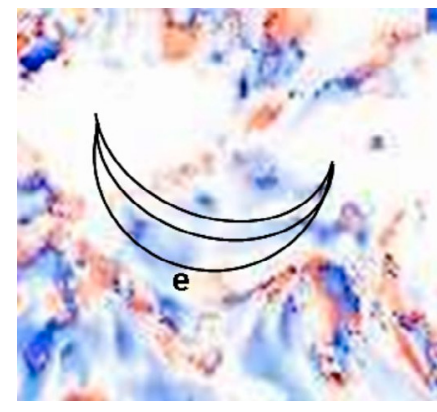

(a)

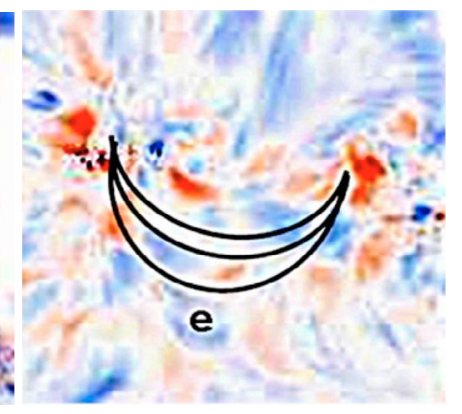

(b)
Fig. 9. Maps of the LOS velocity for the sub-field 2 obtained with the cloud model a) and Doppler shift methods b). Blue (red) areas indicate upward (downward) plasma motions. Black lines outline the analyzed structures.

Table 2. Median of plasma velocity values at the center and footpoints of the AFS $e$.

\begin{tabular}{cccc}
\hline \hline & EF & CP & WF \\
\hline Time & \multicolumn{2}{c}{$\begin{array}{c}\text { Median LOS velocity } \\
\left(\mathrm{km} \mathrm{s}^{-1}\right)\end{array}$} \\
& \multicolumn{3}{c}{$( \pm 1.2)$} \\
\hline 15:52:51 & -2.3 & +1.6 & -2.8 \\
15:53:50 & -1.9 & +1.6 & -2.3 \\
$15: 54: 49$ & -1.7 & +1.5 & -2.2 \\
$15: 55: 48$ & -1.8 & +1.5 & -1.8 \\
15:56:47 & -3.0 & +1.5 & -2.8 \\
$15: 57: 46$ & -1.9 & +1.4 & -2.1 \\
$15: 58: 44$ & -2.1 & +1.5 & -2.5 \\
$15: 59: 43$ & -2.0 & +1.4 & -1.8 \\
\hline
\end{tabular}

We have presented the results obtained by studying morphological and dynamical properties of small-scale chromospheric features observed in the Ca II $854.21 \mathrm{~nm}$ line with IBIS. Recent studies (Cauzzi et al. 2008; Vecchio et al. 2007, 2008; Langangen et al. 2008; Reardon et al. 2009) have pointed out that this line provides interesting information for chromospheric plasma diagnostics. It is a very broad line, which shows the chromosphere at relatively low heights $(\sim 800 \mathrm{~km})$. Its wings give information on the photospheric structures, particularly on reverse granulation, while the core provides significant information on chromospheric aspect (Cauzzi et al. 2008; Choudhary 2001). The chromospheric picture resulting from the Ca II $854.21 \mathrm{~nm}$ images is consistent with those provided by $H_{\alpha}$ data, highly structured by the magnetic fields, with the presence of fibrils and mottles, and represents the true nature of the chromosphere.

The Ca II $854.21 \mathrm{~nm}$ data analyzed in this paper, acquired on October 3, 2006 by IBIS, contain a magnetic bipolar region, which can be considered an intermediate structure between an ephemeral and a short-lived active region.

In our study we selected a sample of mottles, almost straight and well separated from each other, and an AFS.

We used two methods to evaluate the LOS plasma velocity in these structures: the Doppler shift of the line centroid and the cloud model. In the past, the second method was mostly used to analyze $\mathrm{H}_{\alpha}$ data and to extract physical information on structures like mottles, fibrils and arch filaments, considering them as clouds inside an unperturbed atmosphere.

The Doppler shift method was applied to all the structures, while the cloud method was applied only to the mottles and to the central part of the AFS. The footpoints of the AFS, in fact, are located in the stronger magnetic concentrations, where the absorption is not sufficient to get an adequate contrast with the background, and the iterative procedure does not converge. Note that the cloud model has some limitations and that the choice of the background might influence the final results (Tziotziou et al. 2003; Tziotziou 2007).

Using the cloud model procedure, we could determine the behaviour of the Doppler width, optical depth, LOS velocities and source function inside the mottles. The results indicate that the first three parameters peak at values that are slightly lower (by a factor 2-3) than those reported in the literature (Tziotziou et al. 2003; Lee et al. 2000), while the peak value for the source 
function is a factor 3 greater. This difference might be justified by the fact that in previous works the line used to determine the source function was the $H_{\alpha}$ line, while in this work we used the Ca II $854.21 \mathrm{~nm}$ line.

The LOS velocities determinated in the four analyzed mottles do not show a regular behavior. In particular, mottle $a$ shows alternating upward and downward motion occurring in phase in all the examined segments, i.e. in the western footpoint anchored in the negative magnetic flux concentration, in the central part and in the eastern ending point. This behavior holds in the first six sequences examined, while in the last sequences the ET exhibits downwad motions and the other two segments are characterized by upward motion. These results confirm the extremely dynamical behaviour of mottles, already highlighted in previous works (Rouppe van der Voort et al. 2007; De Pontieu et al. 2007b; Tziotziou et al. 2003).

In mottle $b$ the velocities near the endpoints seem to reflect the oscillations of $4 \mathrm{mn}$ found by other authors. Initially, upflow (downflow) motions are recorded in the eastern (western) endpoint, while after about $4 \mathrm{mn}$ the plasma motion seems to reverse. The central part shows both upward and downward motion.

Mottles $c$ and $d$ have positive and negative velocities which change along the whole structure with an apparent irregularity.

We found the same behavior of the LOS velocities with both methods, for all the mottles analyzed. The main difference is that the values deduced with the cloud model are usually higher, by a factor of 2 , than those deduced by the Doppler shifts. This agreement confirms that the irregular behavior of the LOS velocity along the mottles should depend only on their intrinsic characteristics.

Other authors also found an irregular trend of vertical velocities along mottles (e.g. Tziotziou et al. 2003), alternating upward and downward plasma motion, especially in their central body, with a period of about $5 \mathrm{mn}$. De Pontieu et al. (2007) found transverse motion in the direction perpendicular to the mottle axis, with a mean horizontal velocity of about $13 \mathrm{~km} \mathrm{~s}^{-1}$ and Suematsu et al. (2008) found similar oscillations (of 1 to $4 \mathrm{mn}$ ) in a series of analyzed spicules, which could be considered magnetic features closely related to mottles. However, 3D non-LTE modeling of synthetic Ca II $854.21 \mathrm{~nm}$ images (Leenaarts et al. 2009) showed that thin dark elongated structures in the simulated line-core images appear at the interface between upflowing and dowflowing plasma, indicating that these structures appear in sites characterized by very complex dynamics.

Another interesting result of our work concerns the presence of an AFS during the declining phase of the region. AFS are usually observed during the first phases of emergence of active regions, as a consequence of the rising of magnetic flux tubes toward higher atmospheric layers (see van Driel-Gesztelyi 2002, for a review). The LOS velocities evaluated are similar, both in trend and in order of magnitude, to those observed for a short-lived active region (Zuccarello et al. 2005). They show positive velocities (which means upward motion) of about $1.5 \mathrm{~km} \mathrm{~s}^{-1}$ at the top and negative ones (downward motion) of about $-2.0 \mathrm{~km} \mathrm{~s}^{-1}$ near the footpoints, without any asymmetry. During the time interval studied $(8 \mathrm{mn})$ this trend remains almost constant.

However, this AFS is observed during the phase of decay of the region analyzed, that is when the magnetic flux measured in the area of $\sim 170 \times 170 \mathrm{Mm}^{2}$ centered around the region, was decreasing (see Fig. 5) and the knots of opposite polarities, after an initial phase of separation, where approaching each other. In this regard, the appearance of an AFS in this phase of magnetic region evolution is in contrast with current models of active region formation (see, e.g Zuccarello et al. 2009, and references therein).

Acknowledgements. The authors wish to thank the referee for her/his comments and suggestions, which led to a sounder version of the manuscript. This work was supported by the Istituto Nazionale di Astrofisica (INAF), by the Catania University, by the Agenzia Spaziale Italiana (contract I/015/07/0) and by the European Commission through the SOLAIRE Network (MRTN-CT-2006035484).

\section{Appendix A: Cloud model}

The cloud model introduced by Beckers (1964) assumes that: a) the structure is fully separated from the underlying chromosphere, b) the source function, radial velocity, Doppler width, and the absorption coefficient are constant along the line of sight (LOS) and c) the background intensity is the same below the structure and in its surrounding atmosphere. Under these assumptions, the radiative transfer equation can be written as follows:

$I(\Delta \lambda)=I_{0}(\Delta \lambda) \mathrm{e}^{-\tau(\Delta \lambda)}+S\left(1-\mathrm{e}^{-\tau(\Delta \lambda)}\right)$

The first term on the right hand represents the absorption of the incident radiation by the cloud, while the second term represents emission by the cloud itself. The radiative transfer equation can be rewritten in terms of the contrast profile $C(\Delta \lambda)$ :

$$
\begin{aligned}
C(\Delta \lambda) & =\left[I(\Delta \lambda)-I_{0}(\Delta \lambda)\right] / I_{0}(\Delta \lambda) \\
& =\left[S /\left(I_{0}(\Delta \lambda)\right)-1\right] *\left[1-\mathrm{e}^{-\tau(\Delta \lambda)}\right]
\end{aligned}
$$

where $I(\Delta \lambda)$ is the observed profile, $I_{0}(\Delta \lambda)$ is the profile emitted by the background, $S$ the source function, $\tau(\Delta \lambda)$ the optical depth. Usually, a Gaussian wavelength dependence is assumed for the optical depth:

$\tau(\Delta \lambda)=\tau_{0} \mathrm{e}^{-\left(\Delta \lambda-\Delta \lambda_{I}\right) /\left(\Delta \lambda_{\mathrm{D}}\right)^{2}}$

where $\Delta \lambda_{I}=\lambda_{0} v / c$ is the Doppler shift, $\lambda_{0}$ the line center wavelength, $c$ the speed of light, $v$ the LOS velocity, $\Delta \lambda_{\mathrm{D}}$ the Doppler width and $\tau_{0}$ the line center optical depth. The four adjustable parameters of the model are the source function $S$, the Doppler width $\Delta \lambda_{\mathrm{D}}$, the optical thickness $\tau_{0}$ and the LOS velocity $v$.

The computational procedure applied in this study fits the observed contrast profile with a curve that results from an iterative least-square procedure for non linear functions. The procedure is repeated until the differences between computed and observed profiles are minimized (Fig. A.1a) reports an example of such fit. The coefficients of the fitting curve are functions of the free parameters of the model. As background profile we have taken the mean of all profiles inside a quiet region near the structures analyzed (see Fig. A.1b, for an example).

At the beginning of the iteration, initial values for the free parameters are set. We took: $\tau_{0}=1, \Delta \lambda_{\mathrm{D}}=0.03 \mathrm{~nm}, v=0 \mathrm{~km} \mathrm{~s}^{-1}$, given by Tziotziou et al. (2003), and the empirical approximate relation $S=0.155+0.159 \times C(\Delta \lambda=0)$ for the source function $S$.

In our analysis only profiles that have a contrast at the line center lower than 0.3 were considered. This means that we are considering only structures in absorption which appear dark compared to the background intensity. Besides, we considered only the points along the profile in the range $\Delta \lambda= \pm 0.05 \mathrm{~nm}$, because for $\Delta \lambda$ greater than $0.06 \mathrm{~nm}$ we approach the continuum. For a satisfying agreement, we rejected the fits with a $\chi^{2}$ greater than 1 and that lead to parameters that are physically incorrect (negative values for optical thickness, source function, and Doppler width). 

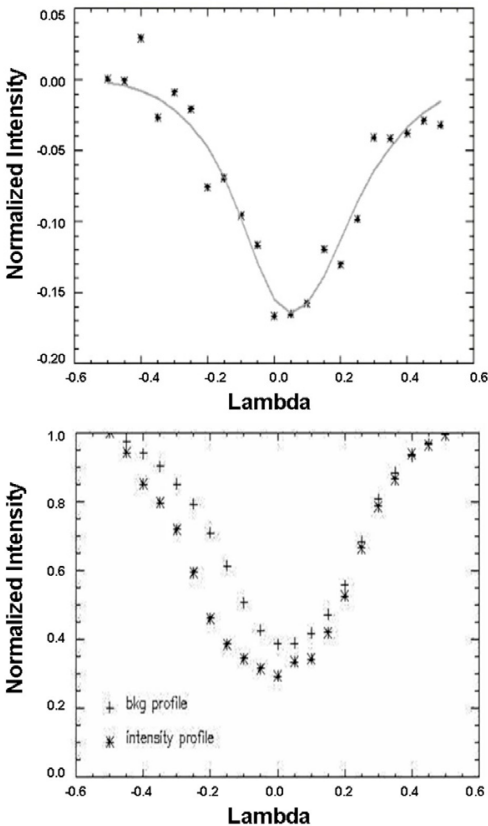

(a)

(b)

Fig. A.1. a) An example of the observed contrast profile (asterisks) with the curve that best fits the profile, b) an example of the background (pluses) and intensity (asterisks) profiles.

\section{References}

Beckers, J. M. 1964, A study of the fine structures in the solar chromosphere, Ph.D. Thesis, Utrecht

Beckers, J. M. 1972, ARA\&A, 10, 72

Berger, M. A., \& Lites, B. W. 2002, Sol. Phys., 213, 213

Cargill, P. J., \& Priest, E. R., 1980, Sol. Phys., 65, 25

Cauzzi, G., Reardon, K. P., Uitenbroek, H., et al. 2008, A\&A, 480, 515

Cavallini, F. 2006, Sol. Phys., 236, 415
Choudhary, D. P., Sakurai, T., \& Venkatakrishnan, P. 2001, ApJ, 560, 439

Christopoulou, E. B., Gorgakilas, A. A., \& Koutchmy, S. 2001, Sol. Phys., 199, 61

Dara-Papamargaritis, H., \& Koutchmy, S. 1983, A\&A, 125, 280

De Pontieu, B., Hansteen, V. H., Rouppe van der Voort, L., van Noort, M., \& Carlsson, M. 2007a, ApJ, 655, 624

De Pontieu, B., Hansteen, V. H., Rouppe van der Voort, L., van Noort, M., \& Carlsson, M. 2007b, ASP Conf. Ser., 368

de Wijn, A. G., \& De Pontieu, B. 2006, A\&A, 460, 309

Freeland, S. L., \& Handy, B. N. 1998, Sol. Phys., 182, 497

Janssen, K., \& Cauzzi, G. 2006, A\&A, 450, 365

Hansteen, V. H., De Pontieu, B., Rouppe van der Voort, L., van Noort, M., \& Carlsson, M. 2006, ApJ, 647, L73

Langangen, O., De Pontieu, B., Carlsoon, M., et al. 2008, ApJ, 679, L167

Lee, C. Y., Chae, J., \& Wang, H. 2000, ApJ, 545, 1124

Leenaarts, J., Carlsson, M., Hansteen, V., et al. 2009, ApJ, 694, 128

Priest, E. R. 1981, in Solar Active Regions. Boulder: Colorado Assoc. Univ. Press, ed. F. Q. Orrall, 213

Reardon, K. P., Uitenbroek, H., \& Cauzzi, G. 2009, A\&A, 500, 1239

Rimmele, T. R. 2004, Proc. SPIE 5490, ed. D. Bonaccini Calia, B. L. Ellerbroek, \& R. Ragazzoni, 34

Rouppe van der Voort, L. H., De Pontieu, B., Hansteen, V. H., Carlsson, M., \& van Noort, M. 2007, ApJ, 660, L169

Shibata, K., Tajia, T., \& Steinolfson, R. S. 1989, ApJ, 345, 584

Spadaro, D., Billotta, S., Contarino, L., Romano, P., \& Zuccarello, F. 2004, A\&A, 425, 309

Suematsu, Y., Wang, H., \& Zirin, H., 1995, ApJ, 450, 411

Suematsu, Y., Ichimoto, K., Ketsukawa, Y., et al. 2008, ASP Conf. Ser., 397

Tsiropoula, G., Alissandrakis, C. E., \& Schmieder, B. 1994, A\&A, 290, 285

Tziotziou, K. 2007, ASP Conf. Ser., 368, 217

Tziotziou, K., Tsiropoula, G., \& Mein, P. 2003, A\&A, 402, 361

Tziotziou, K., Tsiropoula, G., \& Mein, P. 2004, A\&A, 423, 1133

Tziotziou, K., Heinzel, P., \& Tsiropoula, G. 2007, A\&A, 472, 287

van Driel-Gesztelyi, L. 2002, IAU Coll., 188, ESA SP-505, 113

van Noort, M., Rouppe van der Voort, L., \& Lofdahl, M. G. 2005, Solar Phys., 228,191

Vecchio, A., Cauzzi, G., Reardon, K., Janssen, K., \& Rimmele, T. 2007, A\&A, 461, L1

Vecchio, A., Cauzzi, G., \& Reardon 2008, A\&A, 494, 269

Yoshimura, K., \& Kurokawa, H. 1999, ApJ, 517, 964

Zuccarello, F., Battiato, V., Contarino, L., et al. 2005, A\&A, 442, 661

Zuccarello, F., Guglielmino, S. L., Battiato, V., et al. 2009, Acta Geophys., 57, 52 